\title{
Polifarmacoterapia: estratégias de racionalização
}

Paula Broeiro, ${ }^{*}$ Inês Maio, ** Vítor Ramos***

\section{RESUMO}

Introdução: A polifarmacoterapia é um termo recente que tem subjacente a utilização de vários medicamentos em simultâneo. O termo mais utilizado até ao momento tem sido polifarmácia, que pode ter diversos significados e tem uma conotação negativa. A polifarmacoterapia está associada ao aumento do risco de iatrogenia e de gastos em saúde. Nem sempre a polifarmacoterapia é inapropriada e compete ao médico de família a difícil tarefa de aplicar individualmente a melhor evidência disponível. Processo de raciocínio terapêutico: A decisão de prescrever é um processo complexo que tem determinantes prévios e consequências. A formação médica em farmacologia clínica, a indústria farmacêutica e as normas de orientação clínica (que devem reunir a melhor evidência) são os principais determinantes. Existem diversos instrumentos facilitadores do raciocínio terapêutico, dos quais salientamos: as P-drugs, a Lista de Beers (idoso), os suportes electrónicos de apoio à prescrição e a utilização de acrónimos tais como AVOID Mistake, NO TEARS, entre outros.

Estratégias de racionalização da polifarmacoterapia: A abordagem da polifarmacoterapia é uma medida de prevenção quaternária (prevenção da iatrogenia) que pressupõe uma medicina reflexiva e uma decisão partilhada com o doente. É listado um conjunto de etapas, numa sequência lógica, das quais se salienta: a listagem exaustiva de todos os medicamentos (incluindo não sujeitos a receita médica, os fitoterapêuticos, os dietéticos e os alternativos), o conhecimento farmacológico de cada medicamento prescrito, a simplificação dos regimes e explicitação dos objectivos terapêuticos.

Palavras-chave: Polifarmácia; Polifarmacoterapia; Polimedicação; Hiperfarmacoterapia.

\section{INTRODUÇÃO}

1 polifarmácia, ou polimedicação, consiste na utilização simultânea de múltiplos fármacos/medicamentos. ${ }^{1}$ Este conceito tem habitualmente uma conotação negativa porque está, muitas vezes, associado à utilização inapropriada de medicamentos, mais que à sua utilização racional. ${ }^{1,2} \mathrm{~A}$ polifarmácia pode ser apropriada como, por exemplo, na diabetes mellitus. O que importa é avaliar se o perfil da medicação é excessivo ou desnecessário. ${ }^{1} \mathrm{O}$ termo polifarmácia é como um chapéu-de-chuva que cobre: o número excessivo de medicamentos, múltiplas doses e/ou consumo desnecessário. ${ }^{1}$ Recentemente, o termo que melhor reflecte a utilização de múltiplos medicamentos é o de hiperfarmacoterapia. ${ }^{2}$ Em português, o termo anglo-saxónico hiperpharmacotherapynão reflecte o con-

\footnotetext{
*Assistente Graduada de Clínica Geral no Centro de Saúde do Lumiar

**Interna do Internato Médico de Medicina Geral e Familiar no Centro de Saúde da Amora

***Chefe de Serviço de Clínica Geral na USF Marginal (Centro de Saúde de Cascais); Professor convidado da Escola Nacional de Saúde Pública (UNL).
}

ceito, por isso, optámos por polifarmacoterapia.

A combinação de fármacos pode ser benéfica, apropriada e suportada por evidência, em diversas situações clínicas como, por exemplo, a prevenção primária da doença macrovascular na diabetes, que pode justificar o tratamento com um ou mais hipoglicemiantes orais e/ou insulina, um ou mais anti-hipertensores, terapêutica hipolipemiante e ácido acetilsalicílico. Cumprindo as várias normas orientadoras num doente com multimorbilidade (doença pulmonar obstrutiva crónica, diabetes tipo 2, osteoporose, hipertensão arterial e osteoartrose) pode estar recomendado o uso de 12 medicamentos. ${ }^{3}$ Ao médico de família cabe o bom senso de adaptar individualmente, em cada situação, a melhor evidência disponível.

O risco de reacções adversas estimado é de $6 \%$ quando se tomam dois medicamentos em simultâneo e de $50 \%$ quando são administrados cinco medicamentos diferentes. ${ }^{1}$

A prevalência de polifarmacoterapia em Portugal é desconhecida. Na literatura, apenas se encontraram es- 
tudos locais, num dos quais a prevalência de polifarmacoterapia minor (2 a 4 fármacos) foi de $30,6 \%$ e major (5 ou mais fármacos) de $17,2 \%$. Na população idosa, a polifarmacoterapia minor foi $39,6 \%$ e major $37,1 \%{ }^{4}$

A polifarmacoterapia, enquanto problema, é uma co-responsabilidade dos prescritores e dos utentes. $\mathrm{Da}$ parte do médico, os principais contributos para a polifarmacoterapia-problema são a prescrição excessiva e/ou inapropriada, a continuação de tratamentos mesmo depois de deixarem de ser eficazes e a utilização de fármacos para tratar efeitos indesejáveis de outros. $\mathrm{O}$ utente, por sua vez, contribui para a polifarmacoterapia-problema quando: se automedica sem dar conhecimento ao seu médico, toma um medicamento para cada sintoma, visita vários médicos sem informar cada um dos medicamentos que efectua e, por último, utiliza várias farmácias. ${ }^{1} \mathrm{O}$ envolvimento dos farmacêuticos poderá ser uma estratégia útil para lidar adequadamente com o problema. Os médicos e os utentes devem ser educados para o risco do uso inapropriado de medicamentos e de como o farmacêutico pode ser um recurso para reduzir esse risco. ${ }^{5}$

O consumo inapropriado de medicamentos está ainda associado a outros factores, como o número de medicamentos diários, o nível sócio-económico, o sexo feminino e os sistemas de saúde. Os regimes terapêuticos complicados conduzem ao aumento do número de reacções adversas, à má adesão, à falência terapêutica, à pior qualidade de vida e a mais gastos em saúde. ${ }^{1}$

\section{DETERMINANTES DA PRESCRIÇÃO}

Os determinantes da prescrição de medicamentos são: a formação médica básica, os colegas mais velhos (prescrição $a d$-hoc), a influência do marketing farmacêutico, o sistema de prescrição, as normas orientadoras e a medicina baseada na evidência.

\section{Formação médica básica}

Nos jovens médicos, a dificuldade sentida na prescrição de medicamentos tem, pelo menos, duas explicações com origem na formação médica básica. Por um lado, o ensino da farmacologia, centrado no medicamento, é tradicionalmente efectuado numa fase precoce, sem nenhuma ligação à clínica. Por outro lado, nos anos clínicos, a formação tende a centrar-se nos problemas de saúde. ${ }^{6,7}$
Prescrição ad-hoc e influência do marketing farmacêutico A prescrição ad-hoc é efectuada por mimetização de colegas mais experientes ou por influência de estratégias de marketing que enviesa as noções de medicina baseada em evidência. ${ }^{8}$ Os prescritores devem desenvolver um espírito crítico relativamente à influência dos colegas mais experientes e da indústria, na sua prescrição. Os líderes de opinião são estratégias eficazes de promoção de novos medicamentos, em regra médicos pagos pela indústria para repetirem, entusiasticamente, as suas interpretações de resultados de ensaios clínicos. Os sistemas de saúde e os utentes necessitam de médicos que conheçam bem os medicamentos que prescrevem (restringindo o seu arsenal a produtos de uso bem estabelecido) e que prescrevam menos e melhor. ${ }^{8}$

\section{Principais erros de prescrição}

Os erros de prescrição mais frequentes são os erros de distracção e os de distorção. Em farmacoterapia, aproximadamente, três quartos dos erros de prescrição devem-se a erros de distracção. As estratégias para reduzir a distracção incluem a separação do processo cognitivo de prescrever, de tarefas secundárias (ex. interrupções por parte dos utentes). Estes erros de distracção são evitáveis com uma prática reflexiva e minimizados com os sistemas electrónicos de prescrição. Os erros de distorção estão muito associados à prescrição manual (ilegibilidade, incompreensibilidade de símbolos ou abreviaturas) e são passíveis de ultrapassar com a prescrição electrónica. ${ }^{9}$

Os suportes electrónicos de prescrição melhoram a segurança, a qualidade, a eficiência e o custo-efectividade. A prescrição automática permite completar o perfil do medicamento com acesso a alertas que reduzem a prescrição inapropriada (interacções medicamentosas, com problemas de saúde). ${ }^{10}$

\section{Normas de orientação clínica e medicina baseada na evidência}

A evidência robusta é suportada por ensaios clínicos aleatorizados e controlados mas, por vezes, os resultados desses estudos não respondem à necessidade de alguns grupos (ex. idosos, minorias). ${ }^{11}$ A evidência disponível é ainda insuficiente para responder a muitas das dúvidas clínicas e é difícil a sua aplicação individual. ${ }^{12}$ 
O desenvolvimento de normas orientadoras tenta reunir a melhor evidência disponível para os médicos e pacientes. A aproximação da melhor evidência à prática $\mathrm{e}$ a sua implementação são intervenções necessárias. ${ }^{13}$

\section{PROCESSO DE RACIOCÍNIO TERAPÊUTICO}

A prescrição faz parte de um processo de decisão clínica que deverá ser holístico centrado na pessoa. Há uma série de competências necessárias para o acto de prescrever. Assim, a tomada de decisão terapêutica, ou o raciocínio terapêutico, é uma etapa integrada num processo global de decisão clínica que inclui o diagnóstico.

A escolha do tratamento deve basear-se em duas etapas: (1) utilização de normas orientadoras e/ou formulários; (2) adequação da aplicabilidade individual (ex. multimorbilidade e/ou polifarmacoterapia) da melhor

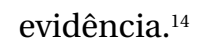

Em geral, utilizam-se dois tipos de raciocínio: um analítico, outro não analítico (Figura 1). O raciocínio analítico, característico dos médicos menos experientes, em geral, é mais lento, consumidor de tempo e, se possível, baseado em evidência. Ao contrário, o raciocínio não analítico é rápido, subconsciente e baseado na experiência. A repetição das situações clínicas faz com que, ao longo do tempo (experiência), se tenha mais frequentemente um raciocínio não analítico, particularmente em casos simples, doença aguda em que são utilizados os fármacos ou tratamentos com os quais o médico está mais familiarizado (P-drugs ou P-treatments) ${ }^{14}$ Em situações mais complexas como a multimorbilidade e a polifarmacoterapia, frequentemente, mesmo médicos experientes têm que utilizar um processo reflexivo (analítico).

\section{INSTRUMENTOS DEAPOIO À DECISÃOTERAPÊUTICA} $\mathrm{P}$ (personal) «drugs»

O guia para a boa prescrição da OMS identifica como uma das estratégias as $\mathrm{P}$-drugs e os $\mathrm{P}$ - treatments e explicita os critérios de selecção. ${ }^{6} \mathrm{O}$ que diferencia uma $\mathrm{P}$-drug de um P-treatment é que este último inclui medidas não farmacológicas.

A escolha de P-drugs baseia-se em critérios de eficácia, segurança, conveniência e custo. São, em geral, medicamentos com que o médico deve ficar familiarizado e são as suas prioridades terapêuticas. A maioria dos

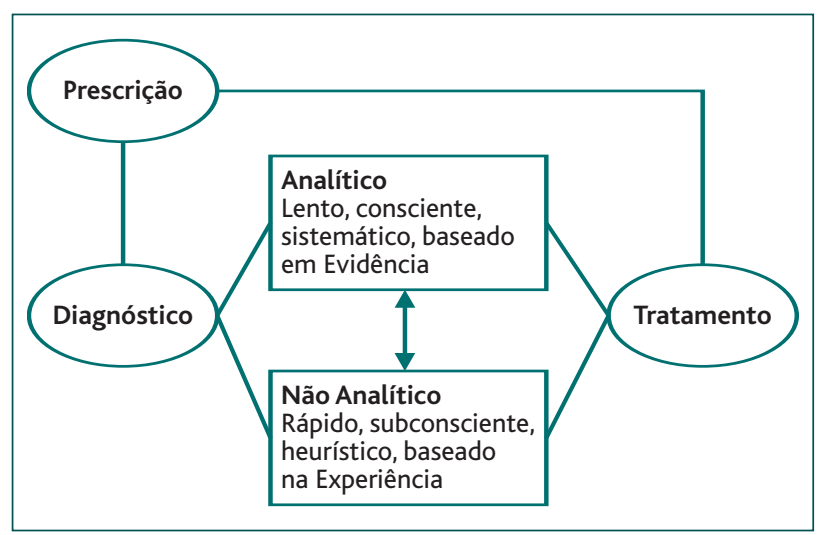

Figura 1. Modelo hipotético de raciocínio terapêutico.

médicos prescreve, regularmente, apenas 40 a 60 medicamentos.

Mesmo utilizando as P-drugs teremos que adaptar o perfil farmacológico a cada doente, por isso, não basta que um medicamento seja efectivo, deve ser conveniente (contributo para a adesão à terapêutica). ${ }^{6}$

A maior parte dos medicamentos que surgiram depois da década de noventa não trouxe um benefício acrescido em relação aos medicamentos mais antigos, menos caros e com riscos melhor conhecidos. Os novos produtos são, em geral, novas formulações, inovações farmacológicas, extensão de indicações, novas vias de administração, em que o investimento em publicidade é desproporcionalmente superior à verdadeira inovação. ${ }^{8}$ Nos medicamentos novos, em geral, são exageradamente antecipados os benefícios e minimizados os riscos conhecidos e não conhecidos. ${ }^{8}$

Como referido, as P-drugs devem incluir o custo. Por isso, o prescritor deve ter sempre em mente que um medicamento vendido com nome genérico é habitualmente mais barato que o produto original. Entre dois medicamentos do mesmo grupo farmacoterapêutico, devemos considerar o que está há mais tempo no mercado (indicando mais experiência e provavelmente mais segurança) ${ }^{6}$

\section{Prevenir a medicação inapropriada}

(idosos - Lista de BEERS)

Nos idosos, há factores que aumentam a sua vulnerabilidade aos fármacos: farmacocinéticos (diminuição da capacidade funcional renal e hepática, diminuição 


\section{QUADRO I. Medicamentos inapropriados nos idosos - Lista de Beers adaptada ao mercado Português}

Independentemente do Diagnóstico

- Cetorolac

- Citrato de orfenadrina

- Isoxsuprina

- Nitrofurantoina

- Doxazosina

- Testosterona

- Clonidina

- Cimetidina

- Sulfato ferroso $325 \mathrm{mg}$

- Anfetaminas

- Nifedipina de curta duração de acção

- Fluoxetina diária

- Laxantes

- Amiodarona

- AINE Não-COX-selectivos

- Estrogéneos

\section{Considerando o Diagnóstico}

- Benzodiazepinas de longa duração de acção e vários problemas

- Propanolol e DPOC/Asma

- Anticolinérgicos e incontinência de urgência

- Antidepressivos triciclicos com síncope, quedas e incontinência

- Benzodiazepinas de curta e intermédia duração de acção com síncope e quedas

- Clopidogrel, com problemas da coagulação

- Descongestionantes e obstrução do fluxo urinário

- Bloqueadores dos canais de cálcio e obstipação

- Fenilpropanolamina e hipertensão

- Bupropiom e convulsões

- Olanzapina e obesidade

- Metoclopramida e doença de Parkinson

- Antipsicóticos e doença de Parkinson

- Tacrina e doença de Parkinson

- Barbitúricos e défice cognitivo

- Anti-espasmódicos e défice cognitivo

- Relaxantes musculares e défice cognitivo

- Estimulantes do SNC e anorexia, malnutrição e défice cognitivo do volume de distribuição e aumento da massa gorda); farmacodinâmicos (aumento da susceptibilidade aos medicamentos); limitação funcional; deterioração cognitiva; menos recursos económicos e multimorbilidade. ${ }^{15}$

A lista de Beers constitui um instrumento útil na avaliação da qualidade da prescrição nos idosos, porque permite identificar medicamentos potencialmente inapropriados. Esta lista tem sofrido revisões desde 1997, a última das quais foi efectuada em 2002. ${ }^{16}$ Estes critérios devem ser revistos regularmente. No Quadro I encontra-se uma versão adaptada ao mercado português (foram retirados os medicamentos não comercializados).

Em qualquer esquema terapêutico, devem substituir-se os fármacos mais tóxicos por outros de menor toxicidade. A lista de Beers, nos idosos, pode orientar essa decisão.

\section{Acrónimos}

No uso racional dos medicamentos, os acrónimos são frequentemente utilizados para orientar o processo de decisão farmacoterapêutica. Alguns dos utilizados são
QUADRO II. Acrónimo AVOID Mistake

Alergias

Vitaminas e medicamentos naturais

OTC e medicamentos antigos (old)

Interacções

Dependência

M(ENDEL)istake - História familiar e/ou farmacogenómica

(Quadros II e III): AVOID Mistake, protocolo SAIL, NO TEARS e I Can PresCribE ADrug ${ }^{1,17,18}$.

Apesar de não ter sido testado, o protocolo SAIL tem como objectivo a minimização da polifarmacoterapia: Simples, Adverso, Indicação, Lista. ${ }^{1}$

Sendo a prescrição de medicamentos uma tarefa complexa, foi implementado, pelo Departamento de Medicina Familiar e Comunitária do Hospital St Michael's, em Toronto, um programa de formação em farmacoterapia para internos, designado I Can PresCribE A Drug. As linhas de orientação são sobreponíveis aos restantes: I(indicação); C(contra-indicações)an; $\mathbf{P}$ (precauções)res; C(custo,compliance)rib; E(eficácia) 


\section{QUADRO III. Instrumento NO TEARS*}

\begin{tabular}{l|l} 
Necessidade e indicação & $\begin{array}{l}\text { O doente sabe porque toma este medicamento? Necessita dele? } \\
\text { Por quanto tempo? A dose é adequada? Confirma-se o diagnóstico? } \\
\text { Não é melhor um tratamento não farmacológico? }\end{array}$ \\
\hline Open (perguntas abertas) & $\begin{array}{l}\text { Tem algum problema? Vamos acordar o que tomar regularmente? } \\
\text { Pensa que os seus comprimidos funcionam? }\end{array}$ \\
\hline Evidência e guidelines & $\begin{array}{l}\text { Controlar a doença e verificar se existe algum problema subtratado? } \\
\text { Monitorizar o tratamento segundo as recomendações? }\end{array}$ \\
\hline Adverso (efeitos adversos) & $\begin{array}{l}\text { Alterou-se a evidência desde que foi iniciado este tratamento? De acordo com } \\
\text { as recomendações, há algum medicamento inapropriado? A dose é a correcta? }\end{array}$ \\
\hline Redução do risco e prevenção & $\begin{array}{l}\text { Tem algum efeito indesejável? Está a fazer outros medicamentos, incluindo alternativos? } \\
\text { Há interacções, duplicações, contra-indicações e medicamentos para tratar efeitos } \\
\text { indesejáveis de outros? }\end{array}$ \\
\hline Simplificação e conveniência & Existe risco e qual? O medicamento foi ajustado para reduzir o risco? \\
\hline
\end{tabular}

*Adaptado de Lewis T. Using the NO TEARS tool for medication review. BMJ 2004; 329: 434. ${ }^{17}$

QUADRO IV. Links de acesso a informação sobre medicamentos

\begin{tabular}{l|l|l}
\multirow{4}{*}{ Agências } & Portuguesa - INFARMED & http://www.infarmed.pt/portal/page/portal/INFARMED \\
\cline { 2 - 3 } & Europeia - EMEA & http://www.emea.europa.eu/ \\
\cline { 2 - 3 } & Americana - FDA & http://www.fda.gov/ \\
\hline \multirow{2}{*}{ Plataformas } & Epocrates & https://online.epocrates.com/home\# \\
\cline { 2 - 3 } & Drug-reference & http://search.medscape.com/drug-reference \\
\hline
\end{tabular}

A(adverso); D(dose, duração, instruções). ${ }^{18}$

De todos os instrumentos, o que consideramos mais abrangente na revisão da terapêutica foi o NO TEARS: Need and indication; Open questions; Tests and monitoring; Evidence and guidelines; Risk redution or prevention; Simplificacion and switches (Quadro II e III). ${ }^{17}$

\section{Suportes electrónicos}

Os suportes electrónicos de registo clínico têm um componente de apoio à prescrição. Em cada sistema, estes instrumentos podem estar mais ou menos desenvolvidos, facilitando a prescrição e a minimização dos erros. Apesar dos benefícios (incluíndo os alertas), podemos necessitar de informação adicional que, neste momento, se encontra acessível gratuitamente na Internet. Sem prejuízo de outros endereços, salientamos alguns que consideramos relevantes (Quadro IV): Agências do Me- dicamentos (INFARMED; EMEA; FDA), plataformas que incluem monografias dos produtos e suportes de avaliação de interacções (DrugCheckers). As páginas na Internet das Agências do Medicamento dão-nos acesso a informação sobre medicamento, no caso particular do INFARMED é disponibilizada informação actualizada sobre alterações de segurança e qualidade. $\mathrm{Na}$ página do INFARMED, salientamos o acesso ao Prontuário Terapêutico e INFOMED (Resumos de Características do Medicamento e Folhetos Informativos).

\section{ESTRATÉGIAS DE RACIONALIZAÇÃO DA POLIFARMACOTERAPIA}

A racionalização da polifarmacoterapia é uma medida de prevenção quaternária (prevenção de iatrogenia). É mais uma das áreas de complexidade da medicina geral e familiar que deve ser integrada na perspectiva biopsi- 
QUADRO V. Racionalização da prescrição $0^{2,15,20-21}$

- Rever o «saco dos medicamentos» e manter um registo actualizado de todos os medicamentos

(não sujeitos a receita médica, dietéticos, fitoterapêuticos e alternativos)

- Identificar todos os medicamentos pela Designação Comum Internacional (DCI)

- Conhecer o perfil dos medicamentos prescritos (P-drugs)

- Assegurar que não há alternativa não farmacológica (P-treatment)

- Certificar que cada medicamento tem indicação adequada (evidência)

- Assegurar que não existe duplicação de medicamentos da mesma classe

- Preferir medicamentos com dupla acção (ex. IECA)

- Especificar e explicitar objectivos terapêuticos (decisão partilhada e empoderamento)

- Suspender medicamentos de benefício desconhecido

- Suspender medicamentos sem indicação clínica

- Substituir fármacos tóxicos por outros mais seguros (Lista de Beers nos idosos)

- Evitar a cascata de prescrição (tratar um efeito indesejável com outro medicamento)

- Procurar utilizar a regra «uma doença, um medicamento, uma vez ao dia»

- Utilizar suportes informáticos para minimizar o erro

- Considerar o custo do medicamento

- Fornecer instruções que incluam indicação, duração e objectivos a atingir com o tratamento

- Rever regularmente os planos terapêuticos

- Coordenar prescrições de diferentes prestadores

- Recomendar a utilização da mesma farmácia

cosocial de um modelo clínico integrado, centrado no doente: aceitar que existem queixas clinicamente não explicáveis; evitar pseudo-diagnósticos e rótulos (que são o primeiro passo para a medicalização); investir na relação médico-doente e envolver o doente na decisão partilhada; tomar decisões baseadas na evidência (adaptada individualmente a cada situação). ${ }^{19}$

A actualização de conhecimentos através de formação contínua (isenta de interesses comerciais) inclui a selecção e a leitura crítica da informação, bem como a adopção de boas práticas médicas: uso de protocolos (diagnósticos e terapêuticos) baseados nas melhores evidências que são dois imperativos éticos. ${ }^{19}$

A medicalização de problemas psicosociais e de alguns sintomas é um dos factores que predispõe à polifarmacoterapia. Uma das estratégias de prevenção da medicalização da saúde passa pela aceitação de que nem todos os sintomas são clinicamente explicáveis. Esta indiferenciação dos problemas pode estar associada com uma fase precoce da sua história natural, sendo importante utilizar o tempo como recurso. Ao rotular todos os problemas apresentados, induzimos a necessidade de prescrever medicamentos. Para além de se aumentar o risco de iatrogenia, pode alterar-se o curso/manifestação natural da doença ou conduzir, ainda, a uma falsa resolução do problema.

No sentido de sistematizar as etapas para a redução da polifarmacoterapia e a utilização racional dos medicamentos, integrou-se a informação de vários autores e listou-se numa sequência lógica (Quadro V).,2,15,20-21

A divulgação destas estratégias tem como objectivo contribuir para uma prescrição mais racional e para reduzir a polifarmacoterapia inapropriada. Não será certamente exequível aplicar em todos os contactos a lista do Quadro V, mas poderá ser utilizada na revisão dos planos terapêuticos, quando é diagnosticado um novo problema de saúde e sempre que sentimos a necessidade de reflectir sobre a prática.

\section{REFERÊNCIAS BIBLIOGRÁFICAS}

1. Turpin C, Dear M, Walters P. Louisiana Drug Utilization Review (LADUR) Education. Polypharmacy: consequences and management. Disponível em:http://rxweb.ulm.edu/pharmacy/oore/2005\%20Summer\%20Edition.pdf [acedido em 31/10/2007].

2. Bushardt RL, Jones KW. Nine key questions to address polypharmacy 
in the elderly. JAAPA 2005 May; 18 (5): 32-7.

3. Hilmer SN. The dilemma of polypharmacy. Aust Prescr 2008 Feb; 31: 2-3. Disponível em: http://www.australianprescriber.com/upload/pdf/articles/930.pdf [acedido em 13/09/2008].

4. Silva P, Luís S, Biscaia A. Polimedicação: um estudo de prevalência nos Centros de Saúde do Lumiar e de Queluz. Rev Port Clin Geral 2004 MaiJun; 20 (3): 323-36.

5. Ballantyne PJ. The role of pharmacists in primary care. BMJ 2007 May 26; 334 (7603): 1066-7.

6. WHO. Guide to good prescribing. Disponível em: http://whqlibdoc.who.int/hq/1994/WHO_DAP_94.11.pdf [acedido em 31/10/2007].

7. Bajcar J, Kennie N, Iglar K. Teaching pharmacotherapeutics to family medicine residents: a curriculum. Can Fam Physician 2008 Apr; 54 84): 549.e1-6 Disponível em: http://www.cfp.ca/cgi/reprint/53/10/1635 [acedido em 26/09/2008].

8. Biron P, Plaisance M, Lévesque P. Pharmas-co-dependence exposed: would it be time to say, "no thanks"? Can Fam Physician 2007 Oct; 53 (10): 1635-7.

9. Teichman PG, Caffee AE. Prescription writing to maximize patient safety. Fam Pract Manag 2002 jul-Aug; 9 (7): 27-30.

10. Tamblyn R, Huang A, Perreault R, Jacques A, Roy D, Hanley J, et al. The medical office of the 21st century (MOXXI): effectiveness of computerized decision-making support in reducing inappropriate prescribing in primary care. CMAJ 2003 Sep 16; 169 (6): 549-56.

11. Aldrich R, Kemp L, Williams JS, Harris E, Simpson S, Wilson A, et al. Using socioeconomic evidence in clinical practice guidelines. BMJ 2003 Nov 29; 327 (7426): 1283-5.

12. Hoffman JR, Wilkes MS, Day FC, Bell DS, Higa JK. The roulette wheel: an aid to informed decision making. PLoS Med 2006 Jun; 3 (6): e137. Disponível em: http://medicine.plosjournals.org/archive/1549-1676/3/6/pdf/ 10.1371_journal.pmed.0030137-L.pdf [acedido em 31/09/2008].

13. Watkins C, Timm A, Gooberman-Hill R, Harvey I, Haines A, Donovan J. Factors affecting feasibility and acceptability of a practice-based edu- cational intervention to support evidence-based prescribing: a qualitative study. Fam Pract 2004 Dec; 21 (6): 661-9.

14. Richir MC, Tichelaar J, Geijteman EC, de Vries T. Teaching clinical pharmacology and therapeutics with an emphasis on the therapeutic reasoning of undergraduate medical students Eur J Clin Pharmacol 2008 Feb; 64 (2): 217-24.

15. Galvão C. O idoso polimedicado: estratégias para melhorar a prescrição. Rev Port Clin Geral 2006 Nov-Dez; 22 (6): 747-52.

16. Fick DM, Cooper JW, Wade WE, Waller JL, Maclean JR, Beers MH. UPdating the Beers criteria for potentially inappropriate medication use in older adults. Arch Intern Med 2003 Dec 8-22; 163 (22): 2716-24.

17. Lewis T. Using the NO TEARS tool for medication review. BMJ 2004 Aug 21; 329 (7463): 434.

18. Bajcar J, Kennie N, Iglar K. Teaching pharmacotherapeutics to family medicine residents: a curriculum. Can Fam Physician 2008 Apr; 54 (4): 549:e1-e6. Disponível em: http://www.pubmedcentral.nih.gov/picrender.fcgi? artid=2294090\&blobtype=pdf [acedido em 26/09/20089.

19. Melo M. A prevenção quaternária contra os excessos da Medicina. Rev Port Clin Geral 2007 Mai-Jun; 23 (3): 289-93.

20. Pollock M, Bazaldua OV, DobbieAE.Appropriate prescribing of medications: an eight-step approach. Am Fam Physician 2007 Jan 15; 75 (2): 231-6.

21. Fillit HM, Futterman $\mathrm{R}$, Orland $\mathrm{BI}$, Chim T, Susnow L, Picariello GP, et al. Polypharmacy management in Medicare managed care: changes in prescribing by primary care physicians resulting from a program promoting medication reviews. Am J Managed Care 1999 May; 5 (5): 587-94.

\author{
Paula Broeiro \\ E-mail: paulabroeiro@iol.pt \\ Inês Maio \\ E-mail: inesmaio@gmail.com \\ Vítor Ramos \\ E-mail:vramos@ensp.unl.pt
}

ENDEREÇOS PARA CORRESPONDÊNCIA

\begin{abstract}
Introduction: The polypharmacotherapy is a recently used term to define the simultaneous use of some drugs. Until now, the mostly used term has been polypharmacy, which has a negative connotation. The negative sense is related with inappropriate drug uses, iatrogenic risk and health costs. The appropriate polypharmacotherapy is a patient-centered approach challenge, and the family doctor should be able to apply individually the best available evidence.

Therapeutic reasoning process: The prescription decision is a complex procedure. The main determinants of drug prescription are: the basic pharmacologic education of the doctor, the pharmaceutical marketing and the guidelines (synthesizing the best evidence). To acquire a correct therapeutic reasoning it is useful to apply some tools such as: P-drugs identification, Beers list (elderly), automated supports to prescription and acronyms (AVOID Mistake, NO TEARS, among others).

Polypharmacotherapy reasoning strategies: The approach of polypharmacotherapy is a quaternary prevention measure which recommends a reflexive medicine with patient participation. Some logic sequence of steps are listed, such as: exhaustive drug listing (including herbals, dietetic, alternatives), pharmacologic knownledge about prescribed drugs, simple regime and explicit therapeutic aims.
\end{abstract}

Keywords: Polypharmacy; Polypharmacotherapy; Polymedication; Hyperpharmacotherapy. 